\title{
Courtship behaviour influences social partner choice in male guppies
}

\author{
Heather L. Auld ${ }^{\mathrm{a}, \mathrm{b}}$ and Jean-Guy J. Godin ${ }^{\mathrm{a}, *}$ \\ ${ }^{a}$ Department of Biology, Carleton University, Ottawa, ON, Canada \\ ${ }^{\mathrm{b}}$ Present address: Coastal Oregon Marine Experiment Station, Oregon State University, \\ Newport, OR, USA \\ *Corresponding author's e-mail address: jgodin@ carleton.ca
}

Received 26 April 2020; initial decision 25 May 2020; revised 7 August 2020; accepted 10 August 2020; published online 15 September 2020

\begin{abstract}
Although male courtship displays have evolved primarily to sexually attract females, they also generate inadvertent public information that potentially reveals the courter's relative sexual attractiveness and the perceived quality and sexual receptivity of the female being courted to nearby eavesdropping male competitors, who in turn may use this information to bias their social partner choices. We tested this hypothesis by first presenting individual eavesdropping male guppies (Poecilia reticulata) the opportunity to simultaneously observe two demonstrator males whose courtship behaviour was manipulated experimentally to differ, following which we tested them for their preference to associate socially with either demonstrator males. Test males preferentially associated with the demonstrator male who they had previously observed courting a female over the other (non-courting) demonstrator. This social association preference was not expressed in the absence of a female to court. Our findings highlight the potential for sexual behaviour influencing male-male social associations in nature.
\end{abstract}

\section{Keywords}

courtship, guppy, Poecilia reticulata, public information, social associations, social partner choice.

\section{Introduction}

In social species, both males and females should socially associate nonrandomly in a manner that increases their overall likelihood of survival and reproductive success (Krause \& Ruxton, 2002; McGlothlin et al., 2010). Multiple interacting factors, including kinship, physical characteristics (e.g., 
body size, sex), social familiarity and(or) behavioural characteristics of potential social partners, can influence with whom individuals associate socially and the frequency and strength of their associations (e.g., Dugatkin \& Sih, 1995; Metcalfe \&Thomson, 1995; Griffiths \& Magurran, 1997; Krause \& Ruxton, 2002; Lusseau, 2007; Croft et al., 2009; Chiyo et al., 2011; Brask et al., 2012, 2019; Kabasakal et al., 2017). Numerous studies have shown that individuals use not only immediate social information, but also information extracted from prior social experiences, to make behavioural decisions, including social partner-choice decisions (reviewed in Danchin et al., 2004; Dall et al., 2005).

Based on sexual and social selection theories (Andersson, 1994; Bateson \& Healy, 2005; McGlothlin et al., 2010), males are expected to socially associate with each other in a manner that maximizes their individual chances of mating. To date, there is only limited evidence in support of this hypothesis (Oh \& Badyaev, 2010; Formica et al., 2011; Callander et al., 2013; Gasparini et al., 2013). The latter studies have mainly focused on secondary sexual physical traits, such as male body size and colour ornamentation, as target traits for males to base their social partner-choice decisions on. To our knowledge, whether behavioural traits associated with individual mating and reproductive success, such as courtship displays (Andersson, 1994; Houde, 1997), influence social partner choice among males is unknown and has yet to be tested experimentally. In addition to being sexually attractive to females, courtship displays directed by a male towards a female also generate inadvertent public information (sensu Danchin et al., 2004) that can reveal his relative sexual attractiveness (Andersson, 1994; Houde, 1997; Kodric-Brown \& Nicoletto, 2001), competitiveness (Guevara-Fiore et al., 2010), condition or health status (Kolluru et al., 2009) and functional fertility (Matthews et al., 1997; Pitcher \& Evans, 2001; Weir \& Grant, 2010), as well as the quality and sexual receptivity of the female being courted (Guevara-Fiore et al., 2010; Godin \& Auld, 2013), to nearby bystanding or eavesdropping males (sensu McGregor \& Peake, 2000). Eavesdroppers may exploit such public information to later bias their social partner choices (Danchin et al., 2004). While there is evidence that adult male fish pay attention to the courtship displays of other males (e.g., Dzieweczynski \& LaMonica, 2016), it remains unknown whether males use such public information to subsequently bias their attraction or avoidance towards, and social associations with, particular conspecific males over others. Fishes have well developed social cognitive 
abilities (Ward et al., 2007) and can recognize prior social partners individually and later behave preferentially towards them based on their past interactions (e.g., Dugatkin \& Alfieri, 1991; Dzieweczynski et al., 2017).

Here, using the Trinidadian guppy (Poecilia reticulata), we tested experimentally the hypothesis that spatial proximity or social associations among individual males in a population can be mediated by their observations of each other's recent past courtship effort. The guppy is a highly suitable model species to address this hypothesis. It is a sexually dimorphic, non-territorial and promiscuous live bearing fish that exhibits mutual mate choice (Houde, 1997; Godin \& Auld, 2013). Compared with other vertebrate species, male guppies court females at relatively high rates in nature (Godin, 1995; Houde, 1997). Individual males vary widely and consistently in their courtship effort (Houde, 1997; Ojanguren \& Magurran, 2004; Guevara-Fiore et al., 2010; Head et al., 2010; Godin \& Auld, 2013), which can be plastic and responsive to variation in ecological (e.g., Godin, 1995; Kolluru et al., 2009; Head et al., 2010) and social (e.g., Auld et al., 2015) factors. Sexually-receptive female guppies tend to prefer males that exhibit high courtship display rates towards them, when controlling for other male traits that can influence female choice (e.g., Houde, 1997; Kodric-Brown \& Nicoletto, 2001). In the wild, guppies live in fission-fusion societies comprised of highly dynamic, mixed-sex shoals of individuals who are non-randomly assorted within social networks (Croft et al., 2004, 2012; Brask et al., 2019). Therefore, males within shoals have frequent opportunities to observe each other's behaviour (including courtship displays) and, as such, gain social information which they can subsequently use to make social partner-choice and mating decisions.

Studies testing whether or not male guppies associate with each other based on their relative sexual attractiveness or sexual competitiveness have focused on physical characteristics, namely body length and (or) coloration (Gasparini et al., 2013; Auge et al., 2016), as target traits. The results of the latter studies are mixed, such that in some populations male guppies will associate with other conspecific males based on their phenotypes, but in different ways depending on ambient social and ecological contexts. Using laboratory-reared guppies originating from a high-predation population in Trinidad, Gasparini et al. (2013) found that males preferentially associated with males who were less sexually attractive (i.e., less colourful) than themselves when in the presence of females. In contrast, using wild guppies from 
the Upper Aripo River, Trinidad (same study population as in the current study), Auge et al. (2016) did not find any evidence for assortative or disassortative male social partner choice based on either body length or colour ornamentation, in either the laboratory or the field. In addition to physical traits, courtship display rate or effort in individual male guppies may potentially mediate social associations among them, as mentioned above (refs. loc. cited).

Using a dichotomous choice paradigm (Auge et al., 2016), we tested individual eavesdropping males for their preference to associate socially with either of two previously-observed paired stimulus males, whose courtship effort (whether they courted a female or not) was manipulated experimentally to differ, but otherwise were matched for body length and overall body colour ornamentation. We tested two alternative predictions based on the comparative evaluation hypothesis (Bateson \& Healy, 2005), which posits that a given male's sexual attractiveness and competitiveness will depend on the others with whom he is being compared rather than being a function of his own absolute qualities. If male guppies are selected to maximize their relative sexual attractiveness to females and mating success, then focal individuals should socially associate preferentially with the non-courting (presumably less attractive and less competitive) stimulus male over the paired courting one, whom they had previously observed. Alternatively, if males are selected to increase their encounter rate and thus mating opportunities with sexuallyreceptive females, then focal males are expected to socially associate preferentially with the courting stimulus male over the paired non-courting one.

\section{Materials and methods}

\subsection{Study subjects and their maintenance}

The guppies used in our study were laboratory-born descendants of wild adults collected haphazardly by hand seining from the Upper Aripo River (Naranjo tributary), Trinidad, West Indies $\left(10^{\circ} 41^{\prime} 70^{\prime \prime} \mathrm{N}, 61^{\circ} 14^{\prime} 40^{\prime \prime} \mathrm{W}\right)$, a low-predation population (Houde, 1997; Magurran, 2005). The reproductive cycle of adult female guppies is approximately 3-4 weeks, from egg fertilization until the birth of young or parturition (Houde, 1997). Females are sexually receptive only as virgins and for a short period (3-4 days) following parturition in post-partum females (Houde, 1997). To ensure the availability 
of sexually-receptive virgin females for our experiment, the fish were separated from each other as juveniles as soon as males showed development of the gonopodium. The fish were held in single-sex aquaria filled with filtered aged tap water $\left(24-26^{\circ} \mathrm{C}\right)$ and illuminated overhead with fluorescent lighting on a $13 \mathrm{~h} \mathrm{~L}$ : $11 \mathrm{~h} \mathrm{D}$ illumination cycle for at least one month prior to experimentation. Female and male aquaria were placed in alternating order on shelving to ensure that all fish had visual exposure to multiple members of the opposite sex. They were fed ad libitum 2-3 times daily with commercial flake food (Nutrafin ${ }^{\mathrm{TM}}$ ) and live brine shrimp nauplii (Artemia franciscana).

Because individual familiarity can influence social associations in the guppy (Griffiths \& Magurran, 1997), the fish used in each behavioural trial were selected from different non-adjacent stock aquaria and were presumably socially unfamiliar with each other prior to testing.

\subsection{Experimental apparatus}

Our experiment comprised three staged social-context treatments, carried out in random sequence, each with two paired consecutive phases.

\subsubsection{Phase 1 (viewing phase)}

For this first phase of the experiment, the experimental apparatus consisted of a clear Plexiglas test aquarium $(40 \times 20 \times 25 \mathrm{~cm}$; $\mathrm{L} \times \mathrm{W} \times \mathrm{H}$; Figure 1a), containing a focal test male, placed inside a water bath $(100 \times 100 \times 30 \mathrm{~cm})$. The walls of the water bath were covered with white plastic sheets to provide a uniform visual background. Water was $15 \mathrm{~cm}$ deep and maintained at 24$26^{\circ} \mathrm{C}$. Five smaller, similar-sized and clear Plexiglas containers (each $10 \times$ $13 \times 20 \mathrm{~cm}$ ) were placed adjacent to one another in a single row along one of the sides of the water bath and facing the larger viewing aquarium at a distance of $20 \mathrm{~cm}$ (Figure 1a). A single narrow and perforated Plexiglas compartment $(4 \times 4 \times 20 \mathrm{~cm})$ was placed inside the small container located at each end of the row. An opaque Plexiglas screen in front of each of the latter two end containers obstructed the focal male's view into these containers. A larger, removable opaque Plexiglas could be placed in front of the test aquarium, temporarily blocking the focal male's view of all the small containers (Figure 1a) until the onset of a trial. The entire apparatus was placed inside a blind and illuminated overhead by fluorescent (full spectrum) light tubes. The water bath, the viewing aquarium and all of the smaller containers contained a thin layer of light-coloured gravel on their bottom. 
(a)

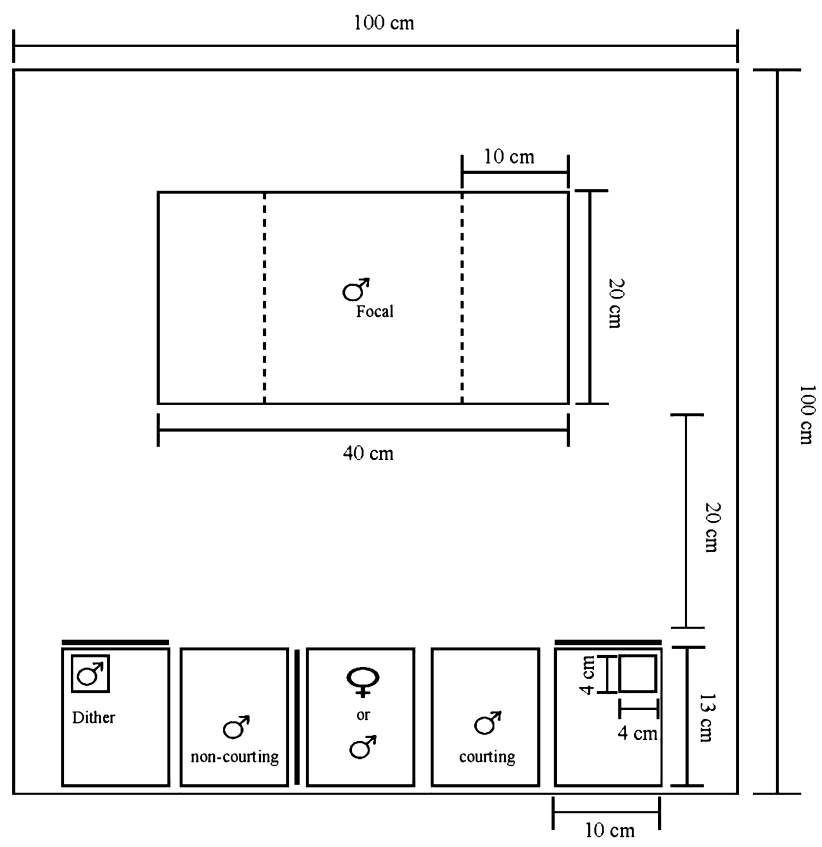

(b)

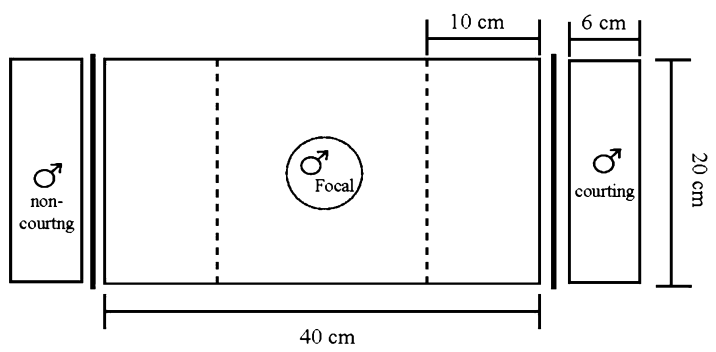

Figure 1. (a) Schematic top view of the experimental apparatus used in the viewing phase of the experiment. The dashed lines represent $10-\mathrm{cm}$ viewing zones directly in line with the small containers containing the two demonstrator stimulus males. The central small container held a stimulus female or male depending on the treatment. (b) Schematic top view of the experimental apparatus used in the partner-choice phase. The central test aquarium held the focal male and the end containers held the paired 'viewing' and 'non-viewing' demonstrator stimulus males. The dashed lines represent $10-\mathrm{cm}$ (social association) preference zones, and the dotted circle denotes the removable clear Plexiglas cylinder that temporarily held the focal male. The thick solid lines in both panels denote removable opaque Plexiglas screens. Here, for example, the figure illustrates the social context for the courtship treatment, wherein the viewing demonstrator male is the 'courting' male and the non-viewing demonstrator male is the 'non-courting' male. 


\subsubsection{Phase 2 (social partner-choice phase)}

For the second phase of the experiment, the experimental apparatus (Figure 1b) consisted of a clear Plexiglas test aquarium $(40 \times 20 \times 25 \mathrm{~cm} ; \mathrm{L} \times$ $\mathrm{W} \times \mathrm{H})$ flanked by a clear Plexiglas container $(6 \times 20 \times 25 \mathrm{~cm})$ at either end. The back wall of the aquarium and three sides of each of the end containers were covered with light brown paper to provide a uniform visual background. The central aquarium served as the partner-choice arena and held the focal male, and the end containers each held a demonstrator stimulus male (= potential social partner). Removable opaque Plexiglas screens could temporarily block the focal male's view of both stimulus males. Because chemical cues can mediate social associations in fishes (Ward \& Hart, 2003), we prevented any exchange of chemical cues between the focal and stimulus males by placing them in separate clear aquaria or containers in both the viewing and social-partner choice phases, which also ensured that the focal males could only use visual cues when assessing the stimulus males as potential social partners. The entire dichotomous-choice apparatus was placed inside a blind and illuminated overhead with a full-spectrum fluorescent light tube.

\subsection{Experimental procedures}

\subsubsection{Phase 1 (viewing phase)}

For any given behavioural trial, a sexually receptive stimulus female was first selected randomly from a stock aquarium and placed in the central small container of the apparatus (Figure 1a). Next, an eavesdropping focal male was selected from a different stock aquarium and placed in the larger viewing aquarium that faced the stimulus fish (Figure 1a). From a different stock aquarium than the focal male, two other males that were matched in standard body length and colour ornamentation as closely as possible (see Table 1) were then selected to serve as demonstrator stimulus males. One of the paired stimulus males, selected at random, was designated the 'viewing' male and placed in one of the two containers adjacent to the stimulus female (Figure 1a); this male could view and potentially court (through the clear walls of their adjacent Plexiglas containers) the stimulus female located in the central container. The other paired stimulus male was designated the 'non-viewing' male and gently placed in the adjacent container on the other side of the stimulus female, but could not view nor court this female because of an opaque screen blocking his view into the central female container (Figure 1a). The sides on which the viewing and non-viewing males were placed 


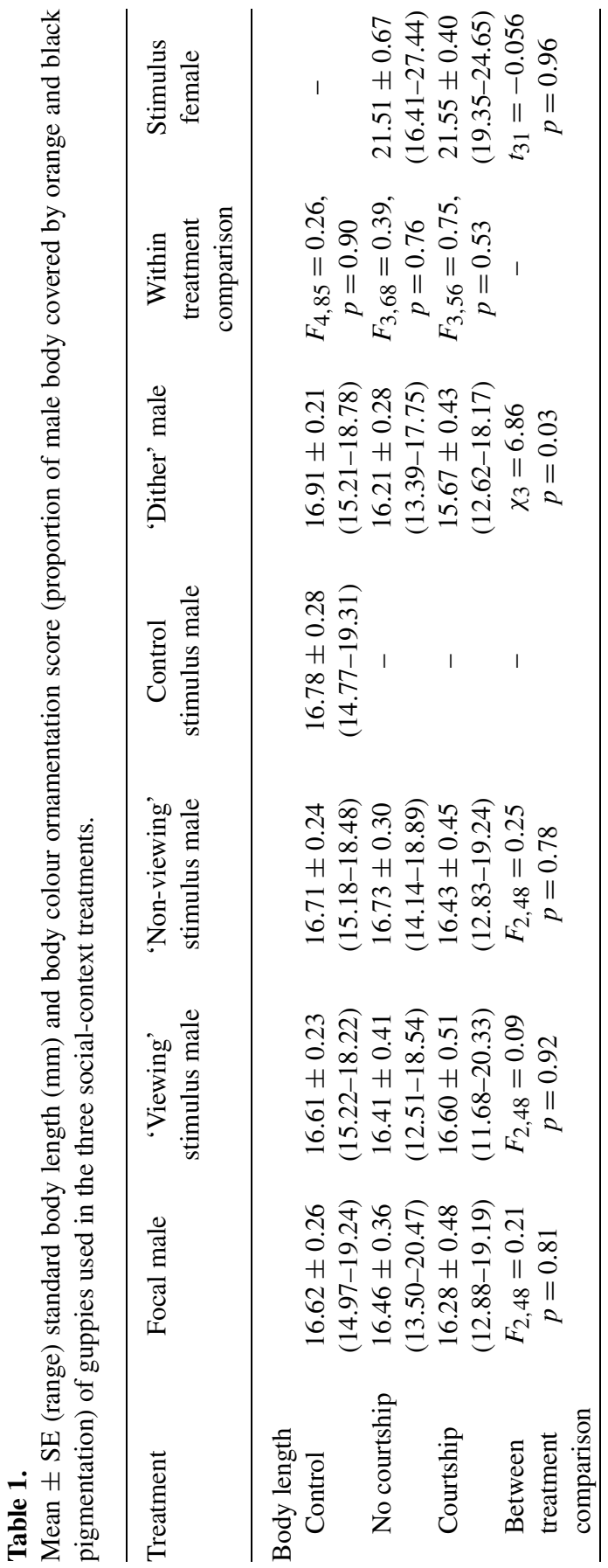




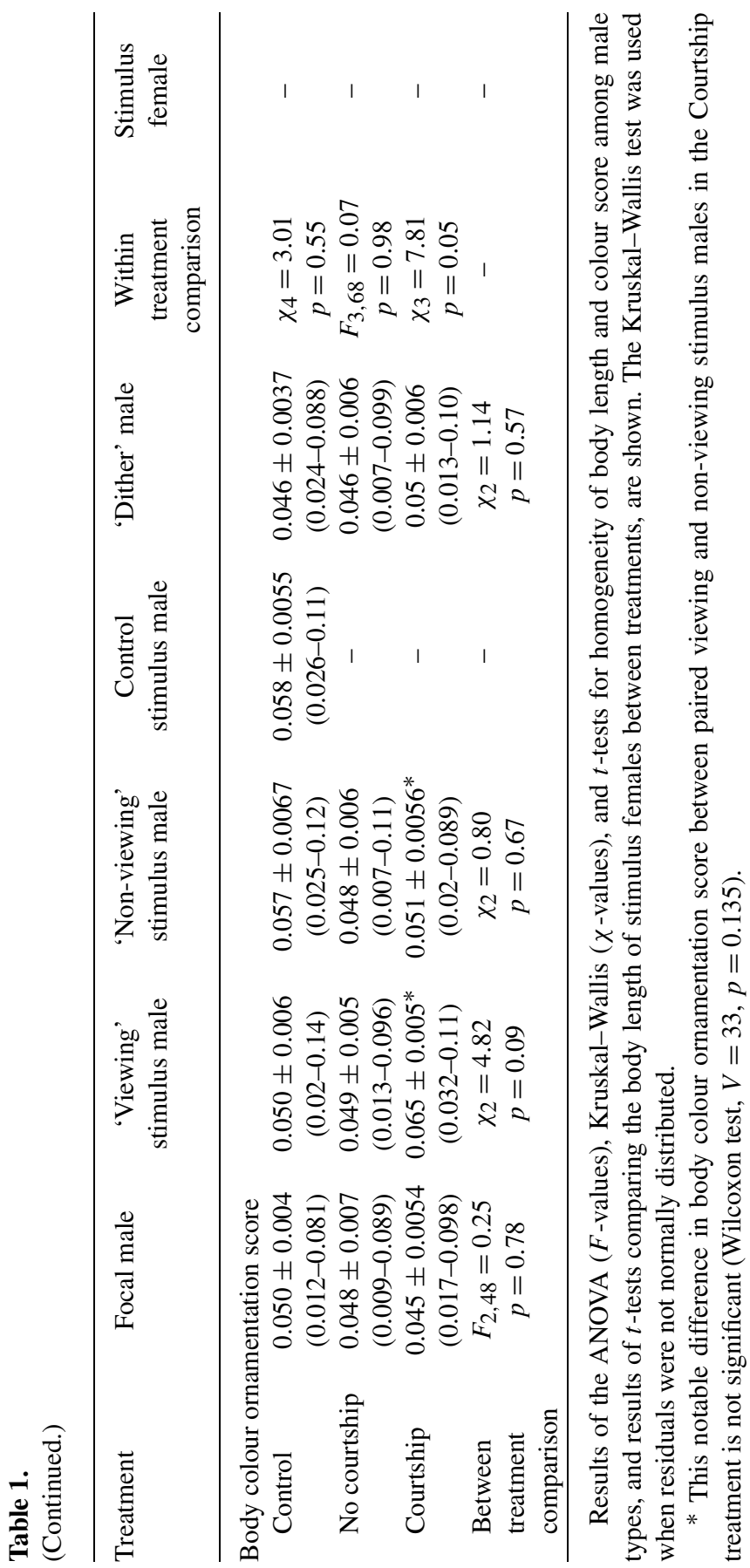


was alternated between trials. In some of the trials, the viewing stimulus male spontaneously courted the female (courtship treatment, $N=15$ ), whereas in other trials the viewing male did not court the female (no courtship treatment, $N=18$ ). In a third treatment (control treatment, $N=18$ ), we controlled for the possibility that males would preferentially associate with either stimulus male irrespective of mating context by replacing the stimulus female with a male in the central container (Figure 1a), thereby removing the sexual mating context and thus any possibility of courtship activity by either demonstrator stimulus male.

For each of these three social-context treatments, we controlled for any effect of potential differences in overall activity between the paired stimulus males, which could otherwise result from the viewing male being able to see another fish nearby (the stimulus female) whilst the non-viewing male could not, by placing a third male (a so-called 'dither' fish; Barlow, 1968) into the far corner of the container located at the end of the row of small containers and adjacent to the non-viewing male. This dither male was not visible to the focal male, the stimulus female nor the viewing male at any time owing to opaque screens placed in front of the dither male's end container and between the non-viewing male's container and the central female container (Figure 1a). This set-up was counter-balanced by placing an empty dither male compartment inside the other far-end container adjacent to the viewing male. Once all fish were in place in their respective aquarium and containers, they were allowed to acclimatize for $5 \mathrm{~min}$ with opaque screens in place temporarily between all five small containers and in front of the viewing aquarium, thus effectively preventing any visual contact between the fish. Following this period, the opaque screens between the stimulus female and the viewing male and between the non-viewing male and the dither stimulus male were removed, and these stimulus fish were allowed to view each other for $5 \mathrm{~min}$. During this period, the opaque screen between the focal male and the stimulus fish remained in place. After this brief period, the focal male was given visual access to the stimulus fish and their behavioural interactions (through the clear walls of their respective containers) by slowly removing the opaque screen in front of his viewing aquarium. The opaque screen between the stimulus female and non-viewing male, as well as the opaque screen in front of the two far end (dither male) containers, remained in place for the duration of the trial (Figure 1a). 
Immediately after the removal of the opaque screen in front of the focal male's viewing aquarium, and once he resumed normal swimming behaviour, a 10-min viewing phase commenced. During this phase, the viewing stimulus male had the opportunity to visually interact with the stimulus female and to perform courtship displays towards her. The non-viewing stimulus male did not have visual access to the stimulus female and thus could not interact with her in any way or perform courtship displays towards her. However, the non-viewing male did have visual access to an adjacent stimulus (dither) male that none of the other fish could see. We recorded with stopwatches from behind the blind the time that the focal male spent inside either of the two end zones of the viewing aquarium and directly facing (i.e., observing) the distant viewing or non-viewing demonstrator stimulus males, respectively. This viewing time was regarded as the time that the focal male spent visually 'assessing' the paired stimulus males from a distance. Concurrently, the behaviour of the stimulus fish was recorded using a Canon VIXIA HF M500 digital video camera, positioned directly above the row of small containers. Upon completion of the viewing phase, the focal male and the non-viewing and viewing stimulus males were gently dip-netted out of their respective aquarium and containers and transferred to the partner-choice test aquarium (Figure 1b). The stimulus female and the dither stimulus male remained in their respective containers until the end of the trial for later photography.

From the video films, we recorded the number of sigmoid courtship displays, if any, exhibited by the viewing demonstrator male during the 10 -min viewing phase. The non-viewing males were never observed exhibiting courtship displays, as expected. During a courtship display, a male typically curves his body in an S-shape posture and vigorously fibrates vertically in front of the female for several seconds (see a video movie (.mov) clip in the Electronic Supplementary Materials, ESM 1 at 10.6084/m9.figshare. 12860930). For each trial, we also recorded separately the time that the viewing and non-viewing demonstrator males each spent within $5 \mathrm{~cm}$ (approx. 3 body lengths) of the central container, which housed either a stimulus female or male (depending on the treatment), in each of the three treatments separately.

\subsubsection{Phase 2 (social partner-choice phase)}

Immediately following his removal from the viewing aquarium and in preparation for Phase 2, the focal male was gently placed in a clear Plexiglas 
cylinder ( $7 \mathrm{~cm}$ diam.) in the centre of the dichotomous choice test apparatus (Figure 1b) and opaque screens were placed between the central test aquarium and the adjacent end containers. The viewing and non-viewing demonstrator stimulus males were then each introduced into one or the other of the two end containers. The particular side (left or right) of the apparatus into which these two males were individually placed was the same side they were on during Phase 1 (viewing phase, Figure 1a).

The fish were then allowed to acclimatize to the apparatus for $5 \mathrm{~min}$, during which they could not see each other. After this period, we gently removed both opaque screens to allow visual contact between the focal male and both stimulus males. We then gently lifted the clear Plexiglas cylinder, allowing the focal male to swim freely in the test aquarium and choose to socially associate with either stimulus males for a 20-min preference test. At the end of first $10 \mathrm{~min}$ of this test period, we replaced the opaque screens and gently dipnetted the focal male and returned him to the central clear Plexiglas cylinder in the middle of the test aquarium. To control for the possibility that the focal male might exhibit a preference (bias) for one side of the aquarium over the other rather than a social preference for a particular stimulus male, we switched the sides of the end containers holding the stimulus males. Once the positions of the end containers had been switched, we removed the opaque screens and lifted the Plexiglas cylinder to release the focal male and to begin the second 10-min half of the preference test. From behind the blind, we recorded using stopwatches the time that the focal male spent within each of the two $10-\mathrm{cm}$ wide end preference zones and facing $\left(0 \pm 90^{\circ}\right)$ either stimulus male for both 10-min halves of the test.

A side bias was defined as a focal male spending more than $80 \%$ of his total association time in the same preference zone (i.e., same side of aquarium), even after the positions of the end containers containing the viewing and non-viewing stimulus males had been switched (cf. Schlupp \& Ryan, 1997). Focal males exhibited a side bias in a total of seven trials, which were discarded and excluded from further analysis.

To calculate an overall social preference score for a focal male, we summed the amount of time that he spent associating with the non-viewing and viewing male separately over both halves of the preference test. Following Auge et al. (2016), we expressed the social association score of the focal male for the viewing stimulus male as the proportion of his total association time spent near $(<10 \mathrm{~cm})$ that stimulus male (i.e., sum of total association 
time spent near viewing male/(sum of total association time near the viewing male + total association time near non-viewing male)). At the end of the preference test, all fish were removed from the apparatus, lightly anesthetized and digitally photographed for later measurement of body length and quantification of male body colour ornamentation (see below).

Although the paired stimulus males in each trial were matched for body length and coloration (Table 1), they possessed individually unique body coloration patterns (Houde, 1997), which presumably allowed focal males to visually distinguish between them. Guppies are able to recognize each other individually using visual cues, including body coloration and behaviour, and to subsequently remember their identity for at least 24 h (e.g., Dugatkin \& Alfieri, 1991; Godin \& Dugatkin, 1996; Hughes et al., 1999). The formation of individual recognition in this species can be very rapid, occurring in a matter of only a few minutes (e.g., Dugatkin \& Alfieri, 1991; Godin \& Dugatkin, 1996; Auld et al., 2017). In general, guppies have good associative learning abilities and memory based on colour cues (e.g., Reader et al., 2003; Bisazza et al., 2014).

\subsection{Measurement of fish body length and ornamentation}

At the end of each behavioural trial, all fish were lightly anaesthetized (with MS-222 at 1:10 000 dilution), placed on a piece of white Plexiglas, and their left side photographed next to a metric ruler using a digital camera under standardized lighting conditions. Following photography, the fish were placed in a container of aerated water to recuperate. From the photographs, we measured the standard body length (to the nearest $0.1 \mathrm{~mm}$ ) and area of the left side of the body (excluding the fins) for all fish, and the body colour ornamentation of all males using Image $J$ (http://rsbweb.nih.gov/ij/; Schneider et al., 2012). We quantified the areas of black and yellow-orange (hereafter orange) pigmentation on the left side of each male's body, excluding the fins, because these colours are sexually selected male traits in several Trinidadian guppy populations (reviewed in Houde, 1997) including our study population (Auld et al., 2016). A male's body colour ornamentation score was expressed as the relative area of body coloration (= (black area + orange area)/total body area) to control for variation in male body size.

\subsection{Animal ethics}

This study was approved by the Carleton University Animal Care Committee (protocol No. 13281) and thus meets the guidelines for the use and care of 
research animals of ASAB/ABS and the Canadian Council on Animal Care and the laws of Canada.

\subsection{Statistical analysis}

We analysed our data using R v. 3.0.3 (R Core Team, 2016). In all linear models testing for a treatment effect on dependent variables (see below), we included the differences in the body length and colour ornamentation score of the paired viewing and non-viewing demonstrator stimulus males (Table 1) as co-variates, because the length and colour phenotypes of stimulus males can influence the behaviour of focal males in choice trials in the Trinidadian guppy (Auld et al., 2017). Residuals of models were normally distributed, as revealed by the Shapiro test and visual inspection of Q-Q plots, unless otherwise noted.

We used paired $t$-tests (two tailed) to test whether the paired demonstrator stimulus males (viewing vs. non-viewing males) differed in the amount of time they spent near $(\leqslant 5 \mathrm{~cm})$ the central container, holding either a conspecific female or male, in the apparatus during the viewing phase (Phase 1) for each of the three treatments separately.

We used one-sample $t$-tests (two tailed) to compare, for each treatment separately, the proportion of time the focal males spent observing (in Phase 1) and socially associating (in Phase 2) with the viewing stimulus male against that expected by chance (i.e., proportion $=0.50$ ). We used linear models, followed by Tukey's test for post-hoc multiple comparison of means, to ascertain separately whether these two behavioural measures differed across treatments. For Phase 2, we compared the absolute time focal males spent in the preference zone of either the viewing or non-viewing stimulus male during the preference test between treatments using the KruskalWallis test, as the residuals of linear models which included absolute times were not normally distributed.

For the courtship treatment, we tested using linear models for any relationship between a focal male's social association score for the viewing stimulus male and (i) the proportion of time he spent previously observing the viewing male and (ii) the courtship display rate of the viewing male during the viewing phase.

Finally, we used linear models to explicitly test separately the potential effects of colour and body length of the focal male and the viewing and non-viewing stimulus males on the focal male's association preference. The 
$\mathrm{R}$ codes for all our linear models and their resultant outputs are available in the Electronic Supplementary Materials (ESM 2 at 10.6084/m9.figshare. 12860930). We provide our original raw data in ESM 3 at 10.6084/m9. figshare.12860930.

\section{Results}

\subsection{Phase 1 (viewing phase)}

During this phase, focal males spent similar amounts of time observing the two demonstrator stimulus males (Figure 2a), although more so for the viewing male than expected by chance in the control treatment $\left(t_{17}=2.29\right.$, $p=0.03$, one-sample $t$-test) but not in the courtship treatment $\left(t_{14}=0.9\right.$, $p=0.38)$ nor the no courtship treatment $\left(t_{17}=-0.22, p=0.83\right)$. Although time spent by the focal males observing the viewing stimulus male did not differ across the three treatments (linear model, $F_{2,46}=0.84, p=0.44$; Figure 2a), it did nonetheless predict (but not strongly so, see $R^{2}$ below) their subsequent social association with that stimulus male (linear model, $t_{1,49}=2.07, R^{2}=0.06, p=0.04$ ) in Phase 2. During Phase 1 , the viewing stimulus male spent significantly more time on average near the central container, which housed either a stimulus female or male depending on the treatment, than the paired non-viewing stimulus male in each of the three treatments (see Figure A1 at 10.6084/m9.figshare.12860930).

\subsection{Phase 2 (social partner-choice phase)}

During this second phase, focal males spent on average $975.2 \pm 19.0 \mathrm{~s}$ (range $=671-1168 \mathrm{~s}$ ) out of a possible $1200 \mathrm{~s}$ in the association zone of either the viewing or non-viewing male, indicating that focal males were strongly attentive to the demonstrator stimulus males. The effect of treatment on the focal males' social association time with the viewing male (Figure 2b) was highly significant (linear model, $F_{2,46}=7.62, p=0.0014$, effect size $\eta_{p}^{2}=0.195$ ). This treatment effect was owing to the focal males similarly avoiding the viewing stimulus male significantly more so than expected by chance when the latter had not courted a female (no courtship treatment, $t_{17}=-4.07, p<0.001$, Figure $2 b$ ) or when he was not seen in the proximity of a female (control treatment, $t_{17}=-2.67, p=0.02$, Figure $2 b$ ), whereas focal males were conversely attracted to, and tended to socially associate, with the viewing male when the latter male had been previously observed 

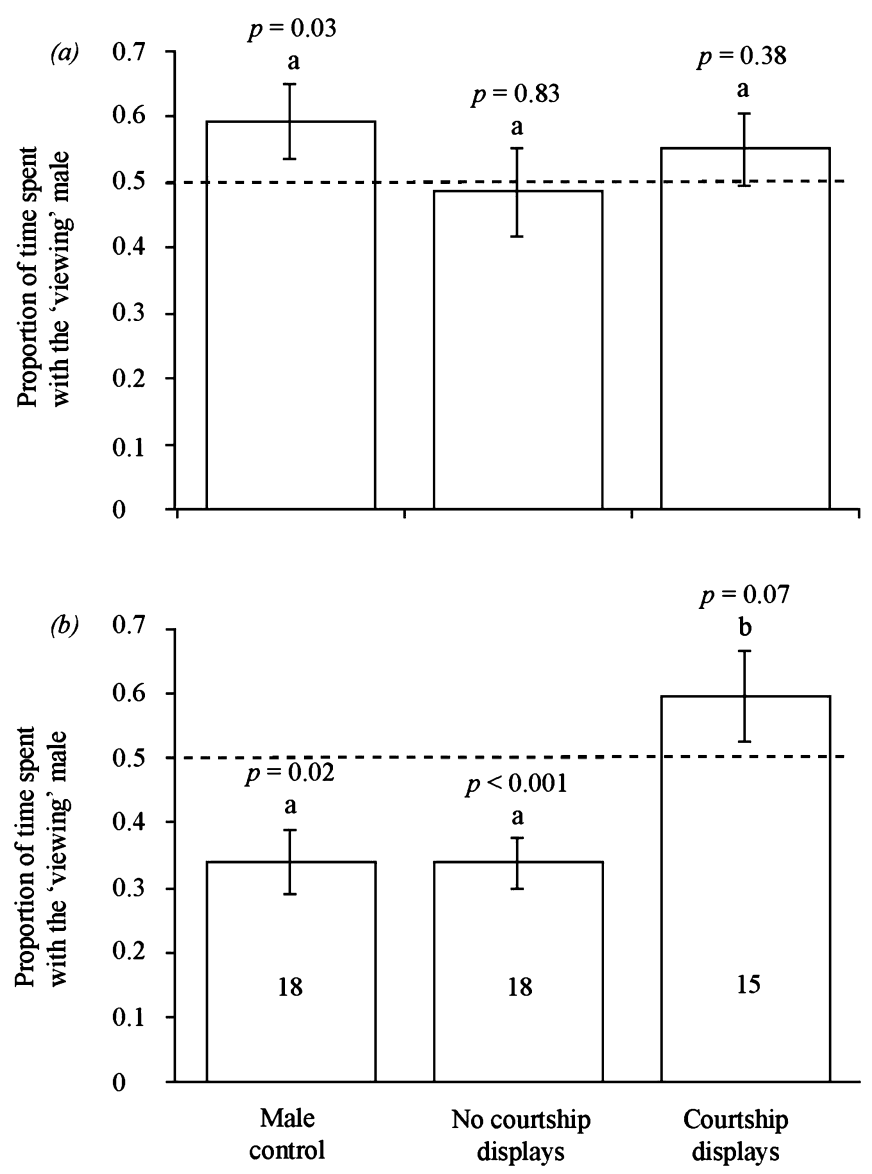

Figure 2. Mean $\pm \mathrm{SE}$ proportion of total viewing or association time the focal male spent near the viewing demonstrator stimulus male in the three treatments during (a) the viewing phase and (b) the social partner-choice phase of the experiment, respectively. The horizontal stippled lines at proportion 0.5 denote no choice (i.e., chance). Histogram bars with similar letters above them are not statistically different from each other $(p>0.05)$, whereas histogram bars with dissimilar letters are significantly different from each other $(p<0.05)$. These multiple-comparison-of-means results were obtained using the Tukey test (panel a) and the Kruskal-Wallis test (panel b). The $p$-value above each bar compares the observed mean value against that expected by chance (one-sample $t$-test). The number of replicate trials for each treatment is shown inside the bars.

courting a female (courtship treatment, $t_{14}=1.98, p=0.07$, Figure $2 \mathrm{~b}$ ). The strength of the focal males' social association preference for the viewing male in the courtship treatment was positively related (linear model, $t_{13}=$ 
2.13, $\left.R^{2}=0.26, p=0.05\right)$ to the latter stimulus male's courtship effort during the prior viewing phase (Phase 1).

Size differences between the paired viewing and non-viewing stimulus males (Table 1) did not affect the social association preferences of focal males (linear model, $t_{2,48}=1.09, p=0.28$ ). Similarly, focal males did not spend a greater proportion of their time with the stimulus male who was more closely matched to himself in length (linear model, $F_{2,48}=0.176$, $R^{2}=0.007, p=0.84$ ) or colour (linear model, $F_{2,48}=1.24, R^{2}=0.05, p=$ $0.30)$. In contrast, differences in body coloration between the paired viewing and non-viewing stimulus males did marginally affect the social association preferences of focal males (linear model, $t_{2,48}=2.31, p=0.03$ ). However, because the paired stimulus males did not differ in their body coloration scores within and between treatments (Table 1), the latter linear model result is unlikely to account for the observed social association preferences of the focal males between treatments (Figure $2 b$ ).

\section{Discussion}

We demonstrated that eavesdropping focal male guppies preferentially associated socially with a demonstrator male who they had previously observed courting a sexually-receptive female over another demonstrator male they had concurrently seen in close proximity to that same female, but could not view nor court her. This social association preference was not expressed by focal males when the demonstrator stimulus male did not court the stimulus female, but otherwise could view and approach her. The strength of the focal male's social association preference for the viewing demonstrator male that courted was positively correlated with both the latter's courtship display rate during the immediately preceding viewing phase and the overall amount of time that he had invested in observing at a distance the viewing demonstrator male (either courting or non-courting) in the latter phase. However, when the viewing demonstrator male did not perform any courtship displays towards the visible stimulus female (i.e., no courtship treatment) or when the female was replaced with another stimulus male (i.e., control treatment), focal males avoided the viewing stimulus male and instead exhibited a strong social association preference for the other (non-viewing) stimulus male. The difference in the observed social association preferences of the focal male between treatments (i.e., male courting vs. not courting during the viewing 
phase) suggests that it was courtship displays per se that socially attracted the focal male towards the courting demonstrator male and not simply the latter male's general behavioural interactions with the stimulus female. Our experimental protocol and design minimized any differences in the time focal males spent observing the paired stimulus males across treatments during the viewing phase and differences in the latter males' body length, colour ornamentation, general activity level and prior social familiarity with each other; therefore, these factors cannot on their own explain the non-random social association preferences reported here.

There are a number of alternative explanations/mechanisms for our main findings summarized above. We discuss below three functional and three proximate alternative explanations/mechanisms. First, at the functional level, if courtship displays render a male more sexually attractive to females (Andersson, 1994; Houde, 1997; Kodric-Brown \& Nicoletto, 2001), then focal male guppies in our study would have been expected to socially associate preferentially with the non-courting (less sexually attractive) stimulus male over the paired courting (more sexually attractive) male in the courtship treatment, so that he might appear relatively more attractive to females, according to the comparative mate evaluation hypothesis (Bateson \& Healy, 2005). This was not the case in our current study, as focal males tended to prefer to associate with the apparently more attractive demonstrator male. Our above finding contrasts those of two previous studies on guppies of different provenance than ours, which reported male social association preferences for apparently less attractive male conspecifics based on prior male proximity to females (Dugatkin \& Sargent, 1994) and male mating preferences for females surrounded by less attractive (drabber) male rivals (Gasparini et al., 2013), respectively. It is plausible that focal male guppies in the current study avoided the non-courting (less sexually attractive) stimulus male in the no courtship treatment because they assessed such non-courting demonstrator males as sexual and sperm competitors rather than female attractors. Consistent with the prudent male under sperm competition hypothesis (Wedell et al., 2002), male Trinidadian guppies, including those from the Upper Aripo River, are sensitive to the presence of nearby rivals and modify their mating tactics in ways that seemingly minimize their risk of sperm competition and maximize their mating success (Jeswiet et al., 2011, 2012; Auld et al., 2015).

Our other finding that male guppies preferred to associate with the nonviewing stimulus male in the male control treatment wherein neither of the 
paired stimulus males exhibited any courtship displays was unexpected. We expected random choice in this treatment. Whilst the containers holding the paired viewing and non-viewing males were placed equidistant from the central container (containing a stimulus male, rather than female, in the control treatment) during the viewing phase, the viewing demonstrator male had visual access to the stimulus fish inside this central container and thus spent more time closer to and 'interacting' with the latter through the clear Plexiglas walls of their respective compartment compared with the paired non-viewing demonstrator male, who did not have visual access to the inside of the central container (see Figure A1 at 10.6084/m9.figshare. 12860930). Because of this asymmetry, the eavesdropping focal male would have observed the viewing demonstrator male in close proximity (i.e., within $5 \mathrm{~cm}$ ) of the stimulus fish within the central container more often than the non-viewing demonstrator male (see Figure A1 at 10.6084/m9.figshare. 12860930). Perhaps a closer proximity of the viewing demonstrator male to the stimulus male in the central compartment (Figure A1 at 10.6084/m9. figshare.12860930) indicated a higher level of competitiveness, which in turn could explain why focal males subsequently avoided the latter demonstrator male and preferred to associate with the non-viewing demonstrator male (i.e., a less competitive male) in the control treatment of the social partner-choice phase.

Second, by preferentially associating with another male that was recently observed attracting and courting a female, an eavesdropping male could socially parasitize his rival's sexual attractiveness to enhance his own mating success through increased frequency of encounters with receptive females, whom he could either court and mate or sneak copulate with (Houde, 1997), and increased opportunities to copy the mate choice of the courting rival male (Auld \& Godin, 2015) compared with searching for females independently, but at the potential cost of an elevated risk of sperm competition (Jeswiet et al., 2011).

Third, ectoparasite-infected male guppies have lower courtship display rates than healthy males who are not infected by ectoparasites (Kolluru et al., 2009). Some fishes avoid social associating with parasitized conspecifics to minimize their individual risk of being infected and(or) predated (reviewed in Krause \& Ruxton, 2002). Moreover, male guppies that are faced with a perceived increase in their ambient predation risk are more fearful and, in response, reduce their conspicuous courtship rate compared to males that 
are less threatened (e.g., Godin, 1995). While it is unlikely that any males in the current experiment were parasitized since they were laboratory born and reared and showed no obvious sign of ectoparasite infection, and there was no threat of predation during the experiment, eavesdropping focal males may nonetheless have assessed the observed difference in courtship activity between the paired demonstrator males as differential parasite load and(or) fear and subsequently avoided the male with the lower courtship rate and presumably associated higher parasite load and(or) fearfulness.

Fourth, at the proximate level, the observed social attraction of eavesdropping focal male guppies towards the viewing stimulus male, who they had previously seen courting a female, may be owing to a sensory bias (Endler, 1992) towards conspecifc males who had been observed actively performing courtship displays. This proposition is supported by our finding of a positive relationship between the number of courtship displays performed by the viewing stimulus male and the strength of the eavesdropping focal male's social association preference for the viewing male.

Fifth, the observed social association bias toward a courting male may not be related to his courtship effort per se, but rather to the increased activity or sensory stimuli associated with courtship displays. Biased attraction towards motion stimuli has been previously documented in fishes (e.g., Krause \& Godin, 1995; Pritchard et al., 2001; Nakayasu \& Watanabe, 2014), Social attraction to the male who was observed displaying higher levels of activity may override any association preference for males who are perceived to be less sexually attractive as a result of differential proximity to a female. Additionally, a reduction in overall mating effort and a further decrease in courtship displays may cause a reduction in the amount of social information available to eavesdropping rivals. Males may prefer social partners who continue to be active sexually and to thereby provide inadvertent social information than to ones who reduce or shut down their sexual behaviour and associated social information in the presence of sexual rivals (cf. Auld et al., 2015). If overt courtship displays are indicative of a male's sexual interest in a female and thus reveal information about the suitability of that female as a mate, then males may seek to associate with other males who inadvertently provide social information about the quality of females by performing overt courtship displays.

Lastly, and related to the above-mentioned sensory bias for motion stimuli, it is also possible that a viewing stimulus male who was excited into 
courting a female during the viewing phase exhibited a higher 'carry-over' level of general activity (movement) than the paired non-viewing and noncourting stimulus male during the immediately following social partnerchoice phase, despite our attempt (using 'dither' males) to minimize any differences in the activity level of these stimulus males during the preceding viewing phase. Such differential motion between the paired stimulus males, if it existed, during the partner-choice phase could have biased the attention and social attraction of focal males towards the stimulus male that they had previously observed courting a female. We cannot discount this simpler alternative explanation for our observation that focal males preferred a previously-observed courting male as a social partner over a non-courting male, because unfortunately we did not video filmed the paired stimulus males during the social partner-choice phase to quantify their respective activity levels.

Prior studies on Upper Aripo River guppies have not found male-male social associations based on body length (Auge et al., 2016; Brask et al., 2019), colour ornamentation (Auge et al., 2016), cooperativeness (Brask et al., 2019) or kinship (Croft et al., 2012; Brask et al., 2019). Therefore, if social partner associations among male guppies occurs in our study population (Upper Aripo River), as it does in other Trinidadian populations (Croft et al., 2012; Brask et al., 2019), it may instead be based on individual spatial proximity to females (Dugatkin \& Sargent, 1994) or on male behaviour such as differential activity (Krause \& Godin, 1995; Pritchard et al., 2001; Nakayasu \& Watanabe, 2014), boldness (Croft et al., 2009), and courtship effort (current study). The occurrence of such non-random social associations based on behaviour could have potential implications for the fitness of individual males and the structure of social networks in nature (e.g., McGlothlin et al., 2010; Oh \& Badyaev, 2010; Silk et al., 2010; McDonald et al., 2013).

In conclusion, our novel findings suggest that male guppies who eavesdrop on the courtship activities of other males are able to remember their identity and subsequently can use information gleaned from their prior courtship behaviour, or lack thereof, to make decisions about which nearby male conspecifics to socially associate with. Irrespective of the underlying mechanism, this highlights the potential for sexual behaviour to mediate male-male social associations in nature. Future studies investigating whether the social association preference of individual male guppies for same-sex conspecifics who they have previously observed courting females, as demon- 
strated in our current study, is a specific response to the latter's courtship displays per se or alternatively to a general attraction toward more active fish, or to another of the above-mentioned plausible alternatives, would provide further insight into the potential mechanism(s) underlying our current results.

\section{Acknowledgements}

We thank two anonymous reviewers for their constructive comments on the manuscript. We are grateful to A.-C. Auge, D. Choudhury, R. Pusiak, V. Scarponi, G. Mazué and S. Potter for assistance with fish care, and R. Pusiak for assistance with the analysis of fish photographs. This research was funded by a Discovery Grant (No. 8074-2010) awarded to J.-G.J.G. from the Natural Sciences and Engineering Research Council of Canada.

\section{References}

Andersson, M. (1994). Sexual selection. — Princeton University Press, Princeton, NJ.

Auge, A.-C., Auld, H.L., Sherratt, T.N. \& Godin, J.-G.J. (2016). Do males form social associations based on sexual attractiveness in a fission-fusion fish society? - PLoS ONE 11: $\mathrm{e} 0151243$.

Auld, H.L. \& Godin, J.-G.J. (2015). Sexual voyeurs and copiers: social copying and the audience effect on male mate choice in the guppy. — Behav. Ecol. Sociobiol. 69: 17951807.

Auld, H.L., Jeswiet, S.B. \& Godin, J.-G.J. (2015). Do male Trinidadian guppies adjust their alternative mating tactics in the presence of a rival male audience? - Behav. Ecol. Sociobiol. 69: 1191-1199.

Auld, H.L., Pusiak, R.J.P. \& Godin, J.-G.J. (2016). Independent mating preferences for male body size and coloration in female Trinidadian guppies. - Ethology 122: 597-608.

Auld, H.L., Ramnarine, I.W. \& Godin, J.-G.J. (2017). Male mate choice in the Trinidadian guppy is influenced by the phenotype of audience sexual rivals. - Behav. Ecol. 28: 362372.

Barlow, G.W. (1968). Dither-a way to reduce undesirable fright behavior in ethological studies. - Z. Tierpsychol. 25: 315-318.

Bateson, M. \& Healy, S.D. (2005). Comparative evaluation and its implications for mate choice. - Trends Ecol. Evol. 20: 659-664.

Bisazza, A., Agrillo, C. \& Lucon-Xiccato, T. (2014). Extensive training extends numerical abilities of guppies. — Anim. Cogn. 17: 1413-1419.

Brask, J.B., Croft, D.P., Thompson, K., Dabelsteen, T. \& Darden, S.K. (2012). Social preferences based on sexual attractiveness: a female strategy to reduce male sexual attention. Proc. R. Soc. Lond. B Biol. 279: 1748-1753. 
Brask, J.B., Croft, D.P., Edenbrow, M., James, R., Bleakley, B.H., Ramnarine, I.W., Heathcote, R.J.P., Tyler, C.R., Hamilton, P.B., Dablesteen, T. \& Darden, S. (2019). Evolution of non-kin cooperation: social assortment by cooperative phenotype in guppies. - Roy. Soc. Open Sci. 6: 181493.

Callander, S., Hayes, C.L., Jennions, M.D. \& Backwell, P.R.Y. (2013). Experimental evidence that immediate neighbors affect male attractiveness. - Behav. Ecol. 24: 730-733.

Chiyo, P.I., Archie, E.A., Hollister-Smith, J.A., Lee, P.C., Poole, J.H., Moss, C.J. \& Alberts, S.C. (2011). Association patterns of African elephants in all-male groups: the role of age and genetic relatedness. - Anim. Behav. 81: 1093-1099.

Croft, D.P., Krause, J., Darden, S.K., Ramnarine, I.W., Faria, J.J. \& James, R. (2009). Behavioural trait assortment in a social network: patterns and implications. - Behav. Ecol. Sociobiol. 63: 1495-1503.

Croft, D.P., Krause, J. \& James, R. (2004). Social networks in the guppy (Poecilia reticulata). — Proc. R. Soc. Lond. B Biol. (Suppl.) 271: S516-S519.

Croft, D.P., Hamilton, P.B., Darden, S.K., Jacoby, D.M.P., James, R., Bettaney, E.M. \& Tyler, C.R. (2012). The role of relatedness in structuring the social network of a wild guppy population. - Oecologia 170: 955-963.

Dall, S.R.X., Giraldeau, L.-A., Olsson, O., McNamara, J.M. \& Stephens, D.W. (2005). Information and its use by animals in evolutionary ecology. — Trends Ecol. Evol. 20: 187-193.

Danchin, E., Giraldeau, L.-A., Valone, T.J. \& Wagner, R.H. (2004). Public information: from nosy neighbors to cultural evolution. - Science 305: 487-491.

Dugatkin, L.A. \& Alfieri, M. (1991). Guppies and the tit-for-tat strategy: preference based on past interactions. - Behav. Ecol. Sociobiol. 28: 243-246.

Dugatkin, L.A. \& Sargent, R.C. (1994). Male-male social association patterns and female proximity in the guppy, Poecilia reticulata. — Behav. Ecol. Sociobiol. 35: 141-145.

Dugatkin, L.A. \& Sih, A. (1995). Behavioral ecology and the study of partner choice. Ethology 99: 265-277.

Dzieweczynski, T.L., Greaney, N.E., Portrais, K.B. \& Stevens, M.A. (2017). I remember you: female Siamese fighting fish recognise prior social partners. - Behaviour 154: 19-35.

Dzieweczynski, T.L. \& LaMonica, H.J. (2016). Court like you mean it: male Siamese fighting fish are less attentive to courting males that have been exposed to an estrogen mimic. Ethology 122: 991-998.

Endler, J.A. (1992). Signals, signal conditions, and the direction of evolution. - Am. Nat. 139: S125-S153.

Formica, V.A., McGlothlin, J.W., Wood, C.W., Augat, M.E., Butterflied, R.E., Barnard, M.E. \& Brodie III, E.D. (2011). Phenotypic assortment mediates the effect of social selection in a wild beetle population. - Evolution 65: 2771-2781.

Gasparini, C., Serena, G. \& Pilastro, A. (2013). Do unattractive friends make you look better? Context-dependent male mating preferences in the guppy. - Proc. R. Soc. Lond. B Biol. 280: 20123072.

Godin, J.-G.J. (1995). Predation risk and alternative mating tactics in male Trinidadian guppies (Poecilia reticulata). — Oecologia 103: 224-229. 
Godin, J.-G.J. \& Auld, H.L. (2013). Covariation and repeatability of male mating effort and mating preferences in a promiscuous fish. — Ecol. Evol. 3: 2020-2029.

Godin, J.-G.J. \& Dugatkin, L.A. (1996). Female mating preference for bold males in the guppy, Poecilia reticulata. — Proc. Natl. Acad. Sci. USA 93: 10262-10267.

Griffiths, S.W. \& Magurran, A.E. (1997). Schooling preferences for familiar fish vary with group size in a wild guppy population. - Proc. R. Soc. Lond. B Biol. 264: 547-551.

Guevara-Fiore, P., Stapley, J. \& Watt, P.J. (2010). Mating effort and female receptivity: how do male guppies decide when to invest in sex? - Behav. Ecol. Sociobiol. 64: 1665-1672.

Head, M.L., Wong, B.B.M. \& Brooks, R. (2010). Sexual display and mate choice in an energetically costly environment. — PLoS ONE 5: e15279.

Houde, A.E. (1997). Sex, color and mate choice in guppies. - Princeton University Press, Princeton.

Hughes, K.A., Du, L., Rodd, F.H. \& Reznick, D.N. (1999). Familiarity leads to female mate preference for novel males in the guppy, Poecilia reticulata. — Anim. Behav. 58: 907-916.

Jeswiet, S.B., Lee-Jenkins, S.S.Y., Ramnarine, I.W. \& Godin, J.-G.J. (2011). Sperm competition risk and mate choice in male Trinidadian guppies, Poecilia reticulata. - Anim. Behav. 81: 639-644.

Jeswiet, S.B., Lee-Jenkins, S.S.Y. \& Godin, J.-G.J. (2012). Concurrent effects of sperm competition and female quality on male mate choice in the Trinidadian guppy (Poecilia reticulata). - Behav. Ecol. 23: 195-200.

Kabasakal, B., Poláček, M., Aslan, A., Hoi, H., Erdoğan, A. \& Griggio, M. (2017). Sexual and non-sexual social preferences in male and female white-eyed bulbuls. — Sci. Rept. 7: 5847.

Kodric-Brown, A. \& Nicoletto, P.F. (2001). Female choice in the guppy (Poecilia reticulata): the interaction between male color and display. — Behav. Ecol. Sociobiol. 50: 346-351.

Kolluru, G.R., Grether, G.F., Dunlop, E. \& South, S.H. (2009). Food availability and parasite infection influence mating tactics in guppies (Poecilia reticulata). - Behav. Ecol. 20: 131-137.

Krause, J. \& Godin, J.-G.J. (1995). Predator preferences for attacking particular prey group sizes: consequences for predator hunting success and prey predation risk. - Anim. Behav. 50: 465-473.

Krause, J. \& Ruxton, G.D. (2002). Living in groups. — Oxford University Press, Oxford.

Lusseau, D. (2007). Evidence for social role in a dolphin social network. — Evol. Ecol. 21: 357-366.

Magurran, A.E. (2005). Evolutionary ecology. The Trinidadian guppy. — Oxford University Press, Oxford.

Matthews, I.M., Evans, J.P. \& Magurran, A.E. (1997). Male display rate reveals ejaculate characteristics in the Trinidad guppy (Poecilia reticulata). - Proc. R. Soc. Lond. B Biol. 264: 695-700.

McDonald, G.C., James, R., Krause, J. \& Pizzari, T. (2013). Sexual networks: measuring sexual selection in structured, polyandrous populations. - Phil. Trans R. Soc. B 368: 20120356. 
McGlothlin, J.W., Moore, A.J., Wolf, J.B. \& Brodie III, E.D. (2010). Interacting phenotypes and the evolutionary process. III. Social evolution. - Evolution 64: 2558-2574.

McGregor, P.K. \& Peake, T.M. (2000). Communication networks: social environments for receiver and signaller behaviour. - Acta Ethol. 2: 71-81.

Metcalfe, N.B. \& Thomson, B.C. (1995). Fish recognize and prefer to shoal with poor competitors. — Proc. R. Soc. Lond. B Biol. 259: 207-210.

Nakayasu, T. \& Watanabe, E. (2014). Biological motion stimuli are attractive to medaka fish. - Anim. Cogn. 17: 559-575.

Oh, K.P. \& Badyaev, A.V. (2010). Structure of social networks in a passerine bird: consequences for sexual selection and the evolution of mating strategies. - Am. Nat. 176: E80-E89.

Ojanguren, A.F. \& Magurran, A.E. (2004). Uncoupling the links between male mating tactics and female attractiveness. — Proc. R. Soc. Lond. B Biol. (Suppl. 6) 271: S427-S429.

Pitcher, T.E. \& Evans, J.P. (2001). Male phenotype and sperm number in the guppy (Poecilia reticulata). — Can. J. Zool. 79: 1891-1896.

Pritchard, V.L., Lawrence, J., Butlin, R.K. \& Krause, J. (2001). Shoal choice in zebrafish, Danio rerio: the influence of shoal size and activity. — Anim. Behav. 62: 1085-1088.

R Core Team (2016). R: a language and environment for statistical computing, version 3.0.3. — R Foundation for Statistical Computing, Vienna, Austria. URL https://www.R-project. org/.

Reader, S.M., Kendall, J.R. \& Laland, K.N. (2003). Social learning of foraging sites and escape routes in wild Trinidadian guppies. — Anim. Behav. 66: 729-739.

Schlupp, I. \& Ryan, M.J. (1997). Male sailfin mollies (Poecilia latipinna) copy the mate choice of other males. - Behav. Ecol. 8: 104-107.

Schneider, C.A., Rasband, W.S. \& Eliceiri, K.W. (2012). NIH image to ImageJ: 25 years of image analysis. — Nat. Meth. 9: 671-675.

Silk, J.B., Beehner, J.C., Bergman, T.J., Crockford, C., Engh, A.L., Moscovice, L.R., Wittig, R.M., Seyfarth, R.M. \& Cheney, D.L. (2010). Strong and consistent social bonds enhance the longevity of female baboons. - Curr. Biol. 20: 1359-1361.

Ward, A.J.W. \& Hart, P.J.B. (2003). The effects of kin and familiarity on interactions between fish. — Fish Fish. 4: 348-358.

Ward, A.J.W., Webster, M.M. \& Hart, P.J.B. (2007). Social recognition in wild fish populations. - Proc. R. Soc. Lond. B Biol. 274: 1071-1077.

Wedell, N., Gage, M.J.G. \& Parker, G.A. (2002). Sperm competition, male prudence and sperm-limited females. — Trends Ecol. Evol. 17: 313-320.

Weir, L.K. \& Grant, J.W.A. (2010). Courtship rate signals fertility in an externally fertilizing fish. - Biol. Lett. 6: 727-731. 